\title{
ASASSN-14ho: Longest-period Dwarf Nova with Multiple Rebrightenings
}

\author{
Taichi KATO ${ }^{*}$ \\ ${ }^{1}$ Department of Astronomy, Kyoto University, Kyoto 606-8502, Japan \\ *E-mail: *tkato@kusastro.kyoto-u.ac.jp \\ Received 201 0; Accepted 2010
}

\begin{abstract}
The post-outburst rebrightening phenomenon in dwarf novae and X-ray novae is still one of the most challenging subjects for theories of accretion disks. It has been widely recognized that post-outburst rebrightenings are a key feature of WZ Sge-type dwarf novae, which predominantly have short $(\lesssim 0.06 \mathrm{~d})$ orbital periods. I found four post-outburst rebrightenings in ASASSN-14ho during its 2014 outburst, whose orbital period has recently measured to be exceptionally long $[0.24315(10) \mathrm{d}]$. Using the formal solution of the radial velocity study in the literature, I discuss the possibility that this object can be an SU UMa-type dwarf nova near the stability border of the 3:1 resonance despite its exceptionally long orbital period. Such objects are considered to be produced if mass transfer occurs after the secondary has undergone significant nuclear evolution and they may be hidden in a significant number among dwarf novae showing multiple post-outburst rebrightenings.
\end{abstract}

Key words: accretion, accretion disks - stars: novae, cataclysmic variables - stars: dwarf novae stars: individual (ASASSN-14ho)

\section{Introduction}

Cataclysmic variables (CVs) are close binaries consisting of a white dwarf (primary) and a mass-transferring red or brown dwarf (secondary). The accreted matter around the primary forms an accretion disk. In some CVs, thermal instability in the accretion disk causes outbursts and these $\mathrm{CVs}$ are called dwarf novae (DNe) [see e.g. Osaki (1996); for general information of cataclysmic variables and dwarf novae, see e.g. Warner (1995)]. SU UMa-type $\mathrm{DNe}$ is a class of DNe showing long and bright outbursts (superoutbursts) during which superhumps with periods a few percent longer than the orbital period $\left(P_{\text {orb }}\right)$ are present. These superhumps are widely believed to be a consequence of tidal instability resulting from the 3:1 resonance in the accretion disk (Whitehurst 1988; Osaki 1989; Hirose and Osaki 1990; Lubow 1991). This 3:1 resonance is considered to be realized only in systems with sufficiently low mass-ratios $\left(q=M_{2} / M_{1}\right.$, where $M_{1}$ and $M_{2}$ are masses of the primary and secondary, respectively).
WZ Sge-type DNe is an extreme extension of SU UMatype DNe with very small $q$, which enables to develop the 2:1 resonance (Lin and Papaloizou 1979; Osaki and Meyer 2002) [see Kato (2015) for a modern review of WZ Sgetype DNe].

One of the defining characteristics of WZ Sge-type $\mathrm{DNe}$ is the frequent presence of post-superoutburst rebrightenings (cf. Kato 2015). These post-superoutburst rebrightenings are also called "echo outbursts" (cf. Patterson et al. 1998). The origin of these postsuperoutburst rebrightenings is not still yet well understood. Some authors claimed that they are a result of enhanced mass-transfer from the secondary (Patterson et al. 2002), while others proposed that they can be understood as the increased turbulence immediately after a superoutburst (Osaki et al. 2001; Meyer and Meyer-Hofmeister 2015) even if the mass-transfer is constant. These has been a numerical simulation which reproduced postsuperoutburst rebrightenings by artificially increasing the 
mass-transfer rate (Buat-Ménard and Hameury 2002). Although this model could reproduce post-superoutburst rebrightenings, the disk shrank in response to the inflow of matter of low specific angular momentum, which is not supported by recent observations of superhump periods in WZ Sge-type DNe (VSNET Collaboration, in prep.).

In the models of Osaki et al. (2001) and Meyer and Meyer-Hofmeister (2015), there is a need for a reservoir of mass in the accretion disk, which enables multiple rebrightenings. Kato et al. (1998) considered that a disk could expand beyond the radius of the 3:1 resonance and that the matter beyond the resonance could play a role of the reservoir. This interpretation appears to be confirmed by the strong infrared excess after the superoutburst (e.g. Uemura et al. 2008) [for a more complete discussion, see Kato 2015]. This interpretation is based on the assumption of an "extra" space beyond the 3:1 resonance, which is only enabled in very low $q$, corresponding to WZ Sgetype DNe.

On the other hand, there have recently been a remarkable advance of understandings of SU UMa-type near the stability border of the 3:1 resonance. A long- $P_{\text {orb }}$ (0.09903 d) dwarf nova V1006 Cyg showed a dip and a post-superoutburst rebrightening (Kato et al. 2016), which had been only recorded in WZ Sge-type DNe. CS Ind $\left(P_{\text {orb }}=0.1242 \mathrm{~d}\right)$ showed a long precursor outburst before a superoutburst, which had been also only known in WZ Sge-type DNe (Kato et al. 2019). These phenomena are considered to be a result of the weak effect of the 3:1 resonance near the stability border of the resonance, leading to premature quenching of the superoutburst and resulting in a considerable amount of matter after the superoutburst as is proposed in WZ Sge-type DNe.

Here I report multiple post-superoutburst rebrightenings in a very long- $P_{\text {orb }}(0.2432 \mathrm{~d})$ in ASASSN-14ho.

\section{ASASSN-14ho}

ASASSN-14ho was discovered by All-Sky Automated Survey for Supernovae (ASAS-SN) Sky Patrol (Shappee et al. 2014; Kochanek et al. 2017) and confirmed as a DN by spectroscopy (Prieto et al. 2014). The outburst started on 2014 September 9, and it did not catch special attention since the phenomenon looked already over at the time of notification in Prieto et al. (2014). Although two rebrightening were already documented in Prieto et al. (2014), the complete picture of the 2014 outburst had been unknown before I obtained the full light curve from the ASAS-SN archive on 2018 October 4 (vsnet-chat 8253). In this vsnet- chat message, ${ }^{1}$ I wrote "The object is too red for a WZ Sge-type dwarf nova. Further study is recommended".

Quite recently, Gasque et al. (2019) reported a radial velocity study of this object and obtained $P_{\text {orb }}$ of $0.24315(10)$ d. From the mass function, Gasque et al. (2019) derived a constraint on the masses of the primary, secondary and the inclination. Their result favored a massive $\left(\sim 1.0 \mathrm{M}_{\odot}\right)$ white dwarf and an undermassive secondary for this $P_{\text {orb }}$

\section{Discussion}

The complete light curve of the 2014 outburst from the ASAS-SN archive is shown in figure 1. The main outburst was followed by four rebrightenings. The $P_{\text {orb }}$ of $0.24315(10) \mathrm{d}$ of ASASSN-14ho is in the range of SS Cygtype DNe (DNe without superhumps or superoutbursts) [see a discussion in Kato et al. (2019) for the borderline between SU UMa-type and SS Cyg-type DNe]. Such multiple rebrightening have been never observed in SS Cygtype DNe.

Given the long $P_{\text {orb}}$, ASASSN-14ho is extremely unlikely a WZ Sge-type DN. Could these rebrightenings be caused by an enhanced mass-transfer in an SS Cyg-type dwarf nova? I consider that they can be interpreted as a phenomenon seen in an SU UMa-type DN near the stability border of the 3:1 resonance, just like V1006 Cyg. The most favorable masses in Gasque et al. (2019) were $M_{1}=1.0 \mathrm{M}_{\odot}$ and $M_{2}=0.28 \mathrm{M}_{\odot}$, respectively, although they were conservative enough to attempt a formal best fit. If I accept them at face value, I obtain $q=0.28$, which indeed close to the stability border of the 3:1 resonance - the modern values being 0.24 based on 3-D numerical simulation (Smith et al. 2007) or 0.33 under condition of reduced mass-transfer (Murray et al. 2000). These value are also consistent with observations of the suggested borderline objects (Kato et al. 2016; Sklyanov et al. 2018).

As for the $q$ value, this object appears to be match perfectly the modern picture of an SU UMa-type DN near the stability border of the 3:1 resonance (confirmation, of course, requires direct detection of superhumps during a future event). Then the next question would be the consistency with the long $P_{\text {orb }}$. Gasque et al. (2019) rather conservatively stated this issue as "the secondary of ASSASN-14ho is somewhat warmer and significantly less massive than their fiducial sequence". It has been already observationally known such a secondary is indeed present (Thorstensen 2015). Although such a result was apparently not present in model parameters in

\footnotetext{
${ }^{1}$ VSNET chat message can be accessed at <http://ooruri.kusastro.kyotou.ac.jp/pipermail/vsnet-chat/>
} 


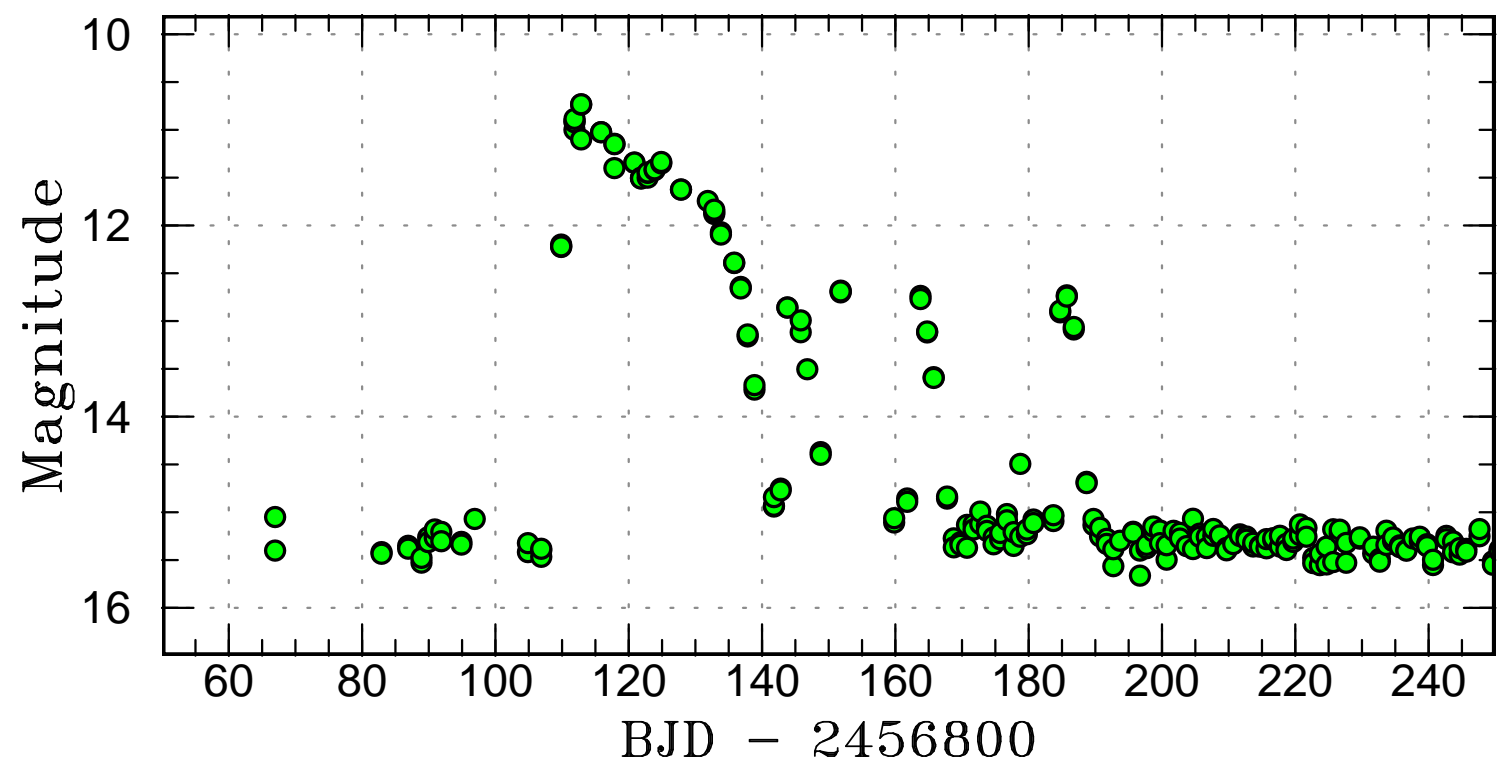

Fig. 1. Light curve of ASASSN-14ho during the 2014 outburst from the ASAS-SN archive. The main outburst was followed by four rebrightenings.

Knigge (2006) as stated in Gasque et al. (2019), studies have shown that such systems can be formed if mass transfer occurs after the secondary has undergone significant nuclear evolution (e.g. Podsiadlowski et al. 2003; Goliasch and Nelson 2015). In particular, the $P_{\text {orb }}-M_{2}$ diagram (figure 6) in Goliasch and Nelson (2015) suggests that systems with $P_{\text {orb }}=5.8 \mathrm{hr}$ and $M_{2}=0.28 \mathrm{M}_{\odot}$ (best values for ASASSN-14ho) should be present as a significant population. In Mróz et al. (2016), two objects were suggested to have long $P_{\text {orb }}$ and multiple rebrightenings [OGLE-BLG-DN-0174 (0.14474(4) d) and OGLE-BLG-DN0595 (0.0972(1) d)]. Although these periods were rather uncertain [see Note added in proof in Kato et al. (2017)], long- $P_{\text {orb }}$ DNe with multiple rebrightenings may not be as rare as previously considered. Further high-cadence wide-field observations would unveil such a population. I must, however, note that final classification requires detection of superhumps and high-cadence observations during the main outburst (before multiple rebrightenings are confirmed) are essential. This is still a challenge even for modern surveys.

\section{Acknowledgments}

The author is particularly grateful to the ASAS-SN team for making their data available to the public.

\section{References}

Buat-Ménard, V., \& Hameury, J.-M. 2002, A\&A, 386, 891
Gasque, L. Claire, Hening, Callum A., Hviding, Raphael E., Thorstensen, John R., Paterson, Kerry, Breytenbach, Hannes, Motsoaledi, Mokhine, \& Woudt, Patrick A. 2019, AJ, 158, 156 Goliasch, J., \& Nelson, L. 2015, ApJ, 809, 80

Hirose, M., \& Osaki, Y. 1990, PASJ, 42, 135

Kato, T. 2015, PASJ, 67, 108

Kato, T., Hambsch, F.-J., Monard, B., Nelson, P., Stubbings, R., \& Starr, P. 2019, PASJ, in press (arXiv / 1909.00910)

Kato, T., Nogami, D., Baba, H., \& Matsumoto, K. 1998, in ASP Conf. Ser. 137, Wild Stars in the Old West, ed. S. Howell, E. Kuulkers, \& C. Woodward (San Francisco: ASP), p. 9

Kato, T., et al. 2016, PASJ, 68, L4

Kato, T., et al. 2017, PASJ, 69, L4

Knigge, C. 2006, MNRAS, 373, 484

Kochanek, C. S., et al. 2017, PASP, 129, 104502

Lin, D. N. C., \& Papaloizou, J. 1979, MNRAS, 186, 799

Lubow, S. H. 1991, ApJ, 381, 259

Meyer, F., \& Meyer-Hofmeister, E. 2015, PASJ, 67, 52

Mróz, P., et al. 2016, ApJS, 222, 9

Murray, J., Warner, B., \& Wickramasinghe, D. 2000, New Astron. Rev., 44, 51

Osaki, Y. 1989, PASJ, 41, 1005

Osaki, Y. 1996, PASP, 108, 39

Osaki, Y., \& Meyer, F. 2002, A\&A, 383, 574

Osaki, Y., Meyer, F., \& Meyer-Hofmeister, E. 2001, A\&A, 370, 488

Patterson, J., et al. 1998, PASP, 110, 1290

Patterson, J., et al. 2002, PASP, 114, 721

Podsiadlowski, Ph., Han, Z., \& Rappaport, S. 2003, MNRAS, 340, 1214

Prieto, J. L., et al. 2014, Astron. Telegram, 6619

Shappee, B. J., et al. 2014, ApJ, 788, 48

Sklyanov, A. S., et al. 2018, Astrophysics, 61, 64

Smith, A. J., Haswell, C. A., Murray, J. R., Truss, M. R., \& Foulkes, S. B. 2007 , MNRAS, 378,785 
Thorstensen, J. R. 2015, PASP, 127, 351

Uemura, M., et al. 2008, PASJ, 60, 227

Warner, B. 1995, Cataclysmic Variable Stars (Cambridge: Cambridge University Press)

Whitehurst, R. 1988, MNRAS, 232, 35 